\title{
Simulation of scaling effects of thermal emission from non-isothermal pixels with the typical three-dimensional structure
}

\author{
L. SU†*, X. LI††, S. LIANG§ and A. H. STRAHLER \\ $\dagger$ Research Center for Remote Sensing and Department of Geography, Beijing \\ Normal University, Beijing 100875, China \\ $\$$ Center for Remote Sensing and Department of Geography, Boston \\ University, Boston, MA 02215, USA \\ $\S$ Department of Geography, University of Maryland, College Park, \\ MD 20742, USA
}

(Received 14 March 2001; in final form 15 July 2002)

\begin{abstract}
This paper suggests approaches to simulate scaling effects of thermal emission from non-isothermal pixels with a typical three-dimensional structure. We simulate effective emissivities of various V-shaped valleys by using our Monte Carlo method under the isothermal assumption. An analytical formula of the effective emissivities is derived based on photon rebounding between surfaces in the valleys. The comparison between the simulated and the analytically modelled effective emissivities shows that the analytical formula is highly accurate. After simulating effective emissivities of the V-shaped valley under several non-isothermal conditions, we find that the structure and component temperature difference of a pixel cause the scaling effects of thermal emission of the pixel. The results, therefore, prove that Planck's law has to be corrected for remote sensing to estimate land surface temperature with high accuracy.
\end{abstract}

\section{Introduction}

Scale is not something new, nor is concerned just by geography, cartography or geographic information science. Scale refers generally to the level of details with which information can be observed, represented, analysed and communicated. However, digital techniques and remote sensing present some fundamental new challenges to this old concept. The importance of scale has been well recognized by the international community of remote sensing and geographic information system (Woodcock and Strahler 1987, Raffy 1994, Quattrochi 1995, Friedl et al. 1995, Quatrochi and Goodchild 1997, Li et al. 1999a, b, Marceau and Hay 2000). Planck's Law is one of the greatest scientific achievements in human history, but its application requires an isothermal blackbody surface. At the scales of the thermal bands of AVHRR (Advanced Very High Resolution Radiometer), Landsat or

\footnotetext{
*e-mail: lihongsu@xinhuanet.com
}

International Journal of Remote Sensing

ISSN 0143-1161 print/ISSN 1366-5901 online (C) 2003 Taylor \& Francis Ltd

http://www.tandf.co.uk/journals

DOI: $10.1080 / 0143116021000023871$ 
MODIS (Moderate Resolution Imaging Spectrometer), land surface is usually heterogeneous in component material, component temperature and three-dimensional structure. These three factors contribute to the scale effects of Planck's Law, resulting in a very confusing problem. Only recently efforts have been made to examine the scale effects of Planck's Law (Becker and Li 1995, Norman and Becker 1995, Wan and Doizer 1996, Li et al. 1999a), where both definitions of temperature and emissivity are evaluated at the pixel scale. Li et al. (1999a) proposed a conceptual model (Li-Strahler-Friedl conceptual model) formally describing the directional emission of heterogeneous, non-isothermal surfaces:

$$
L_{\lambda}(\mu)=\varepsilon_{\text {effective }}(\mu) B_{\lambda}\left(T_{0}\right)
$$

This model yields an effective land surface temperature $T_{0}$ independent of viewing angle and wavelength, and an effective emissivity $\varepsilon_{\text {effective }}(\mu)$ composed of two parts. The first part is a directional emissivity related to the emissivities of individual surface elements present within the sensor's field of view. The second part is an apparent change in emissivity that arises from the temperature frequency distribution of individual surface elements. The conceptual model is then improved by geometric-optical modelling (Li et al. 2000) in a sense that the new model needs only statistic parameters at the pixel scale, without subpixel scale parameters as the Li-Strahler-Friedl conceptual model does. With this improvement, the effective emissivity $\varepsilon_{\text {effective }}(\mu)$ is expressed by geometric-optical scaling-up factor $g(\mu)$. For detailed information about the definitions of the effective land surface temperature $T_{0}$, the effective directional emissivity $g(\mu)$ and derivation of the conceptual model for effective emissivity and scaling-up by the geometric-optical modelling approach, the reader is referred to Li et al. (1999a, 2000).

\section{Simulation on scale effects of thermal emission from isothermal V-shaped valleys}

Under the isothermal condition, the effective emissivity of a three-dimensional pixel depends on its three-dimensional structure (Becker and Li 1995, Norman and Becker 1995, Wan and Doizer 1996; Li et al. 1999a). Su et al. (2000) examined effective emissivity of some cavities by the Monte Carlo method. But their work focused on the hemispherical emissivity, so the directional emissivity has not been studied. Here we investigate how directional variation of effective emissivity depends on the geometry of three-dimensional surfaces by Monte Carlo simulation with one of the simplest cases, the V-shaped valley. The V-shaped valley pixel is a long symmetrical valley perpendicular to the paper, which is the principal plane, and its IFOV (Instantaneous Field of View) just covers the top of the valley. As shown in figure $2, l$ is the top width of the $\mathrm{V}$-shaped valley and $h$ its depth. We assume that $l$ and $h$ are far longer than the wavelength used by earth observing sensors from the visible to the thermal infrared spectrum. In other words, the lengths are from centimetre to kilometre so that the computer simulation experiments are scale independent in optical remote sensing. Moreover, we derive an analytical formula of the effective emissivity for the $\mathrm{V}$-shaped valley, review directional pattern of the effective emissivity relative to the valley, and explain the reasons for the directional pattern. 


\subsection{Monte Carlo algorithm}

Suppose that the width of the $\mathrm{V}$-shaped valley $l$ is one unit (e.g. $1 \mathrm{~m}$ ), its depth is $h$ unit and its material emissivity is 0.96 . Our simulation is based on Kirchhoff's law. In other words, the directional emissivity $\varepsilon(\theta)$ of the valley is obtained through computing its directional-hemispherical reflectance $r(\theta)(\varepsilon(\theta)=1-r(\theta))$.

The simulation is on the principal plane. The transfer of a photon is described as follows:

1. The photon goes down into the $\mathrm{V}$-shaped valley along the zenith from 0 to $85^{\circ}$ with an increment of $1^{\circ}$. The incidence photon traverses openness of the valley randomly according to the incidence direction. Given one point and one direction in the three-dimensional space, the equation of the line, which is the trajectory of the flying photon, can be formed.

2. The photon has to hit one of two slopes in the V-shaped valley. The crosspoint is the solution to the line and slopes equations.

3. If the photon is reflected, its flying direction is determined according to the Lambertian distribution in the slope local coordinate. A new photon line equation is established by the hitting point and the flying direction.

4. If the cross-point falls out of the V-shaped valley, the photon escapes the valley. Otherwise, the photon hits one of two slopes of the valley. Then the simulation goes to step 2. The photon will move according to steps 2 and 4 continuously until it is absorbed or escapes (figure 1).

For $N$ photons we launched in a direction, if $n$ photons escape out of the V-shaped valley, the effective emissivity of the valley at the direction is $1-n / N$. The simulated effective emissivities are shown in figure 2 for the depth $h$ at 1.866, 0.866, 0.5, 0.287 and 0.134 unit, respectively. In figure 2 , the thick black line is for the simulated

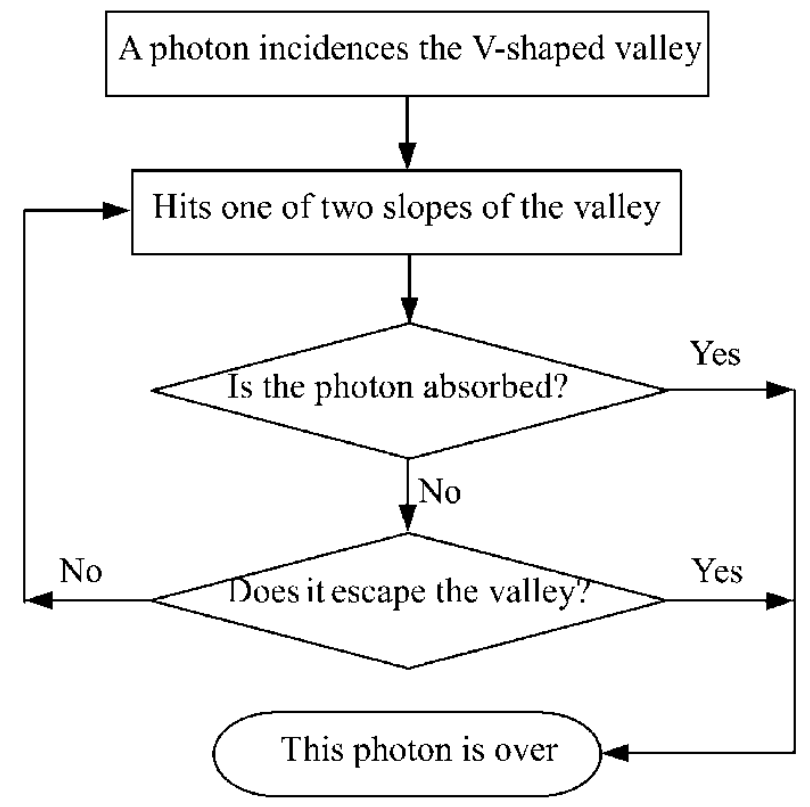

Figure 1. Workflow of tracing a photon. 


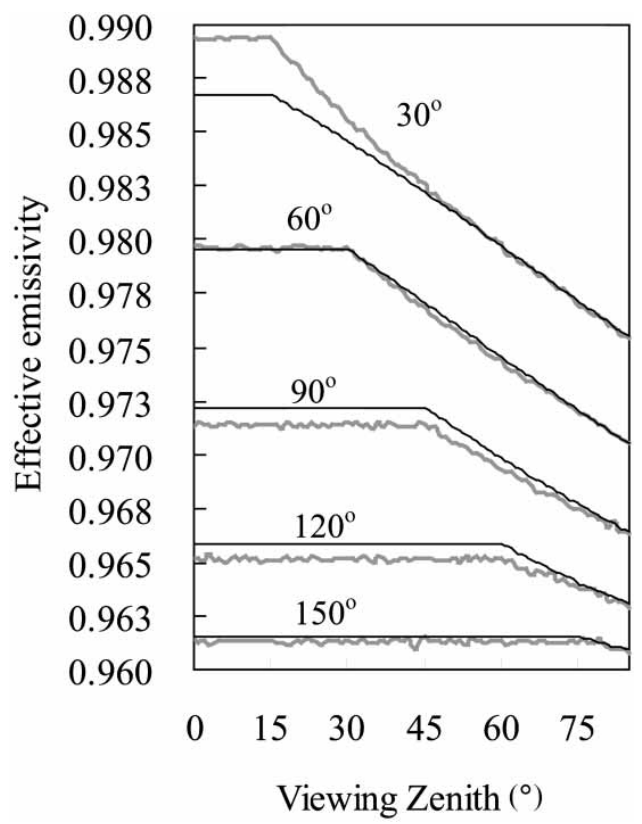

Figure 2. The effective emissivities for various V-shaped valleys.

effective emissivities of the $\mathrm{V}$-shaped valleys, five groups correspond to the bottom angles of $30^{\circ}, 60^{\circ}, 90^{\circ}, 120^{\circ}$ and $150^{\circ}$, and the thin grey one for the modelled.

The effective emissivities of the V-shaped valleys always are greater than their material emissivity. The deeper the valley, the bigger the difference. Moreover, the effective emissivity does not keep constant. When the zenith angle decreases by a few degrees, the effective emissivity increases accordingly. The effective emissivity reaches the maxima as the viewing direction is parallel to the slope. Then effective emissivity holds constant even when the viewing zenith angle increases continuously.

\subsection{Analytically computing effective emissivity}

Figure 3 shows the structure of the $\mathrm{V}$-shaped valley. Its material emissivity is $\varepsilon$, and $\alpha_{V \text { shape }}$ is half of the bottom angle. It is assumed that the parallel incident light goes into the $\mathrm{V}$-shaped valley along the zenith angle $\theta_{\text {viewing viewing on the principle }}$

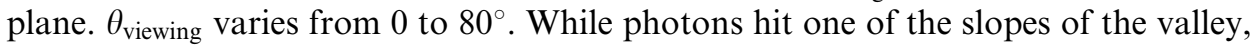
most of them are absorbed, only the $(1-\varepsilon)$ portion is reflected. The reflected photons obey the Lambertian distribution in the local coordinate of the bevel edge. Here we define an escape plane, which is determined by the hitting point on one slope and the top edge of the opposite slope in the valley, and an intercepting angle $\theta_{\text {stop }}$ between the escape plane and the hit slope (see figure 3). The photons reflected over the escape plane will escape the V-shaped valley, displayed by the agency of the big grey arrowhead. The photons under the escape plane will hit the opposite slope of the valley, represented by the small grey arrowhead. It is evident that the probability of photon escaping the valley should be only the function of $\theta_{\text {stop }}$. The probability is called escape probability $P_{\text {escape }}\left(\theta_{\text {stop }}\right) . \theta_{\text {stop }}$ is fixed by the shape of the valley and the hitting position on the slope together. When the hitting position 


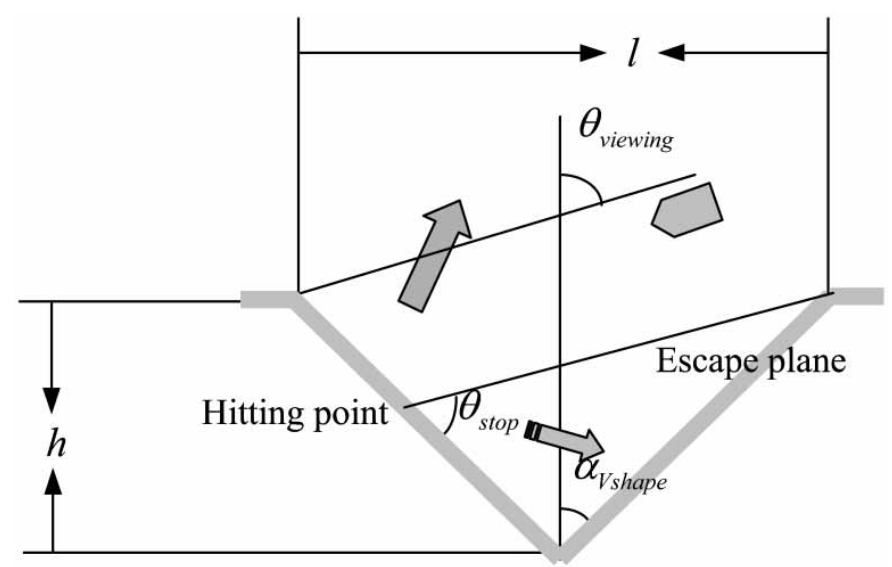

Figure 3. The movement of photons in the V-shaped valley.

changes from the top edge to the bottom edge of the valley, $\theta_{\text {stop }}$ increases monotonously from $\pi / 2-\alpha_{V \text { shape }}$ to $\pi-2 \alpha_{V \text { shape, }}$, the valid range of $\theta_{\text {stop }}$ is changed along with the configuration of the $\mathrm{V}$-shaped valley. It is difficult to derive an analytical expression for $P_{\text {escape }}\left(\theta_{\text {stop }}\right)$ directly, but relatively easy to compute the probability using the Monte Carlo method. Given that the normal of the escape plane is outward from the mouth of the V-shaped valley, if the dot-product of a flying direction and the normal is equal to or greater than zero, the photon escapes out of the valley, otherwise, it falls into the hitting range. The curve of $P_{\text {escape }}\left(\theta_{\text {stop }}\right)$ is shown in figure 3. Apparently, the function is nonlinear. When $\alpha_{V \text { shape }}$ of the valley equals $15^{\circ}, 30^{\circ}, 45^{\circ}, 60^{\circ}$ and $75^{\circ}$, the valid range of the corresponding $\theta_{\text {stop }}$ is $75^{\circ}-150^{\circ}, 60^{\circ}-120^{\circ}, 45^{\circ}-190^{\circ}, 30^{\circ}-60^{\circ}$ and $15^{\circ}-30^{\circ}$, respectively (figure 4).

The parallel incidence light goes through a $\mathrm{V}$-shaped valley along with $\theta_{\text {viewing }}$ uniformly, and hits one of the slopes in the valley. When $\theta_{\text {viewing }}$ is greater than $\alpha_{V \text { shape }}$, the valid range of $\theta_{\text {stop }}$ is from $\pi / 2-\alpha_{V \text { shape }}$ to $\left(\pi / 2-\alpha_{V \text { shape }}\right)+\left(\pi / 2-\theta_{\text {viewing }}\right)$. The photons hit any position of the slope within the valid range of $\theta_{\text {stop }}$ with an

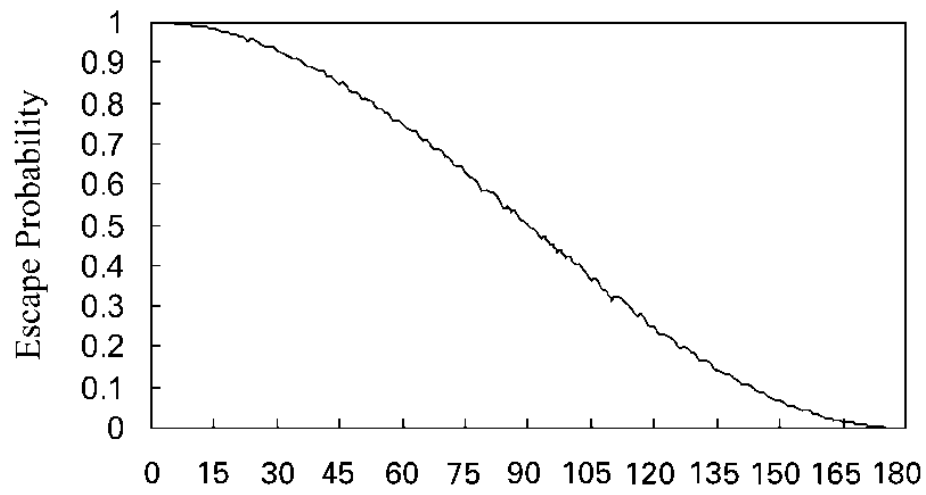

Stop Angle $\left({ }^{\circ}\right)$

Figure 4. The curve showing $P_{\text {escape }}\left(\theta_{\text {stop }}\right)$ changing with $\theta_{\text {stop }}$. 
equal probability, due to the uniform incidence light. Thus, the openness of the V-shaped valley for the slope when it is partially lit, called $K_{\text {open }}^{\theta_{\text {viewing }}}$, is defined as the mean of the escape probabilities of all hitting points for the incidence direction $\theta_{\text {viewing: }}$

$$
K_{\text {open }}^{\theta_{\text {viewing }}}=\int_{\pi / 2-\alpha_{\text {vshape }}}^{\left(\pi / 2-\alpha_{\text {vshape }}\right)+\left(\pi / 2-\theta_{\text {viewing }}\right)} P_{\text {escape }}(\theta) \mathrm{d} \theta /\left(\pi / 2-\theta_{\text {viewing }}\right)
$$

When $\theta_{\text {viewing }}$ is equal to $\alpha_{\text {Vshape }}$, one slope is fully lit. The openness of the slope, called $K_{\text {open }}^{\mathrm{V}}$, can also be calculated by (2).

When $\theta_{\text {viewing }}$ is less than $\alpha_{\text {Vshape }}$, two slopes of the $V$-shaped valley are lit. But the openness of the valley does not increase. The reason is that since the valley is symmetrical, the openness of one slope is equal to each other, i.e. $K_{\text {open }}^{\mathrm{V}}$. As a result of uniform incidence of the parallel light, without loss of generality, we may assume that $1 / m$ part photons hit one slope, $1-1 / m$ part photons hit another, here $m$ is a random number. So the openness of the valley is $1 / m K_{\text {open }}^{\mathrm{V}}+(1-1 / m) K_{\text {open }}^{\mathrm{V}}=K_{\text {open }}^{\mathrm{V}}$ in this case. So far, we have obtained the openness of the valley for all cases.

The analytical formula for the effective emissivity of the V-shaped valley can be derived based on $K_{\text {open }}^{\theta_{\text {viewing }}}$ and $K_{\text {open }}^{\mathrm{V}}$. Generally, it is assumed that the parallel light goes into the $\mathrm{V}$-shaped valley in the direction $\theta_{\text {viewing. }}$ Given material emissivity $\varepsilon$ of the valley, its material reflectance is $\alpha=1-\varepsilon$. In other words, the incidence energy will be absorbed by $(1-\alpha)$, and reflected by $\alpha$ by the valley. $K_{\text {open }}^{\theta_{\text {viewing }}} \alpha$ will escape the valley, and $\left(1-K_{\text {open }}^{\theta_{\text {view }}}\right) \alpha$ will hit the valley again. Now we assume approximately that the $\left(1-K_{\text {open }}^{\theta_{\text {viewing }}}\right) \alpha$ reflected photons are projected on the whole opposite slope of the valley with equal probability. Because the openness of the reflected light is $K_{\text {open }}^{\mathrm{V}}$, when the incidence photons are rebounded secondly, $\left(1-K_{\text {open }}^{\theta_{\text {viewing }}}\right) \alpha(1-\alpha)$ is absorbed, $\left(1-K_{\text {open }}^{\theta_{\text {viewg }}}\right) \alpha \alpha$ reflected, $\left(1-K_{\text {open }}^{\theta_{\text {viewing }}}\right) \alpha^{2} K_{\text {open }}^{\mathrm{V}}$ escapes from the valley, $\left(1-K_{\text {open }}^{\theta_{\text {viewing }}}\right) \alpha^{2}\left(1-K_{\text {open }}^{\mathrm{V}}\right)$ hits the valley again. Analogically, when the incidence photons are rebounded for three times, $\left(1-K_{\text {open }}^{\theta_{\text {viewing }}}\right) \alpha^{2}\left(1-K_{\text {open }}^{\mathrm{V}}\right)(1-\alpha)$ is absorbed, etc. For the photons multiply rebounding in the $\mathrm{V}$-shaped valley, those absorbed can be calculated by the geometric progression:

$$
\begin{aligned}
f_{\text {absorption }}= & (1-\alpha)+\left(1-K_{\text {open }}^{\theta_{\text {viewing }}}\right) \alpha(1-\alpha) \\
& +\left(1-K_{\text {open }}^{\theta_{\text {viewing }}}\right) \alpha^{2}\left(1-K_{\text {open }}^{\mathrm{V}}\right)(1-\alpha)+\ldots
\end{aligned}
$$

Its sum is:

$$
f_{\text {absorption }}=(1-\alpha)+\frac{1+\alpha\left(K_{\text {open }}^{\mathrm{V}}-K_{\text {open }}^{\theta_{\text {viewing }}}\right)}{1-\left(1-K_{\text {open }}^{\mathrm{V}}\right) \alpha}
$$

When $\theta_{\text {viewing }}$ is less than $\alpha_{\text {Vshape }}$, (4) degenerates as follows:

$$
f_{\text {absorption }}=\frac{1-\alpha}{1-\left(1-K_{\text {open }}^{\mathrm{V}}\right) \alpha}
$$

In fact, it is the same formula for photons multiply rebounding between surfaces developed by Li et al. (1996).

The analytical model does a better job for a sallow V-shaped valley than that of a deep valley, as shown in figure 2. The main reason is that the shallower a $\mathrm{V}$-shaped valley the smaller errors introduced by integrating $K_{\text {open }}^{\theta_{\text {viewing }}}$ and $K_{\text {open }}^{\mathrm{V}}$ 
because $P_{\text {escape }}\left(\theta_{\text {stop }}\right)$ becomes smaller in the valid range of $\theta_{\text {stop. Thus, our }}$ approximation will enjoy higher accuracy in the shallow valley. The same conclusion can be reached as we examine the directional pattern of the effective emissivity of a deep V-shaped valley, such as the one when the bottom angle is $30^{\circ}$. When $\theta_{\text {viewing }}$ increases gradually, the valid range of $\theta_{\text {stop }}$ for $K_{\text {open }}^{\theta_{\text {viewin }}}$ decreased accordingly, and the changing scope of $P_{\text {escape }}\left(\theta_{\text {stop }}\right)$ also decreases accordingly. As a result, we compute the escaping part of photons by using the average value $\left(K_{\text {open }}^{\theta_{\text {viewing }}}\right)$ of whole light instead of $P_{\text {escape }}\left(\theta_{\text {stop }}\right)$ of single photon with smaller errors.

The calculated emissivity is bigger than the simulated one for the V-shaped valley with $30^{\circ}$ bottom angle, however, the calculated emissivity is smaller than the simulated one for the $\mathrm{V}$-shaped valley with $150^{\circ}$ bottom angle. That may be explained by the fact that the photons rebounded by one slope of the valley are not scattered equally on the opposite slope. In other words, the scattering position depends on both the photon hitting position and the configuration of the V-shaped valley. Firstly, the photons whose hitting positions near to the bottom of the valley have a smaller probability of escaping the valley. Secondly, the more rebounded photons will encounter the lower part of the opposite slope for a deep valley, and the more rebounded photons will encounter the upper part of the opposite slope for a shallow valley. So the assumption that the rebounded photons are equally scattered on space will overestimate the absorption of a deep valley, and underestimate the absorption of a shallow valley. After all, the calculated emissivity is close to the simulated one. The maximum error of emissivity is less than 0.003 , which causes the error of the temperature estimation by about $0.2 \mathrm{~K}$ under most realistic conditions.

\section{Simulation on scaling effects of thermal emission from non-isothermal V-shaped valleys}

The effective emissivities of non-isothermal surfaces depend on pixel structure, component emissivities and component temperatures. Here is a case study also on the V-shaped valley. The computer simulation approach is used to investigate the contributions of pixel structure, component emissivities and component temperatures on the directional patterns of emissivities.

\subsection{Monte Carlo algorithm}

We assume that the V-shaped valley is open with $l$ metres, unlimited length, and deepness with $h$ metres, and symmetrical configuration. The principal plane is the cross-section of the $\mathrm{V}$-shaped valley, the azimuth at right slope is $0^{\circ}$, and the one at left slope is $180^{\circ}$. The material emissivities of both slopes are $T_{\text {left }}$ and $T_{\text {right }}$, respectively. Their temperatures are $T_{\text {left }}$ and $T_{\text {right }}$, respectively. In order to maintain generality, the thermal radiance is calculated at the wavelength of $10 \mu \mathrm{m}$.

1. Compute the number of photons eradiated by the right slope and left slope of the valley, and the proportion from the left slope, $p_{\text {left }}$. The energy of one photon is $Q_{0}=h v=h c / \lambda \mathrm{J}$, here $h$ is the Planck's constant $\left(6.626 \times 10^{-34} \mathrm{Js}\right), v$ is the frequency $(1 / \mathrm{s}), \lambda$ is the wavelength $(\mathrm{m}), c$ is the velocity of light $\left(3 \times 10^{8} \mathrm{~m} \mathrm{~s}^{-1}\right)$. Given a wavelength $\lambda(\mathrm{m})$, a temperature $T(\mathrm{~K})$ and an emissivity $\varepsilon$, we can get the energy $M(\mathrm{~J})$ from the unit surface area $\left(\mathrm{m}^{2}\right)$, unit time interval $(\mathrm{s})$, unit wavelength interval (m) at wavelength $\lambda$ in upper hemisphere from any body. If $Q_{0}$ is known, the number of photons $N_{\text {photon }}$ can be calculated. The $p_{\text {left }}$ is the proportion of photons emitted by the left slope in total photons emitted by both slopes. 
2. Generate an even random number $r$, if $r<p_{\text {left }}$, the left slope of the valley emits one photon, otherwise, the right slope emits one photon. Generate an even random number again, which is used to find a point on the slope as the sending point of the photon. Flying direction of the photon can be calculated in the local coordinate of the slope according to Lambert's law, and the direction is transformed into the global coordinate of the V-shaped valley. Given one point and one direction, the line equation of the flying photon can be generated.

3. Calculate the crosspoint of the line equation of the photon and the plane equation of the slope. If the crosspoint falls out of the range of the slope, it escapes from the V-shaped valley, otherwise, hitting happens. Record the zenith and azimuth angles of the escaping photon.

4. The reflected photon gets its flying direction in the local coordinate of the slope according to Lambertian law. The direction then is transformed into the global coordinate of the $\mathrm{V}$-shaped valley. The photon moves continuously according to steps 3 and 4 until it escapes from the V-shaped valley or is absorbed by the valley (figure 5). Then the next photon begins to run from step 2 until all photons are processed. After one simulation is finished, count the number of photons at every zenith and azimuth angle to obtain the energy distribution on the $2 \pi$ upper hemisphere. So we can compute directional brightness temperature $T_{\text {pixelbright }}(\mu)$ at every direction $\mu$ on the upper hemisphere.

5. Average directional brightness temperature from $85^{\circ}$ to $95^{\circ}$ on azimuth and from $175^{\circ}$ to $185^{\circ}$ on azimuth at the principal plane as the temperature of the principal plane of the valley, $T_{\text {pixelbright }}(\mu)$. Given $T_{\text {pixelbright }}(\mu)$ and the pixel reference temperature $T_{0}, p(\mu)$ can be computed by the following formula

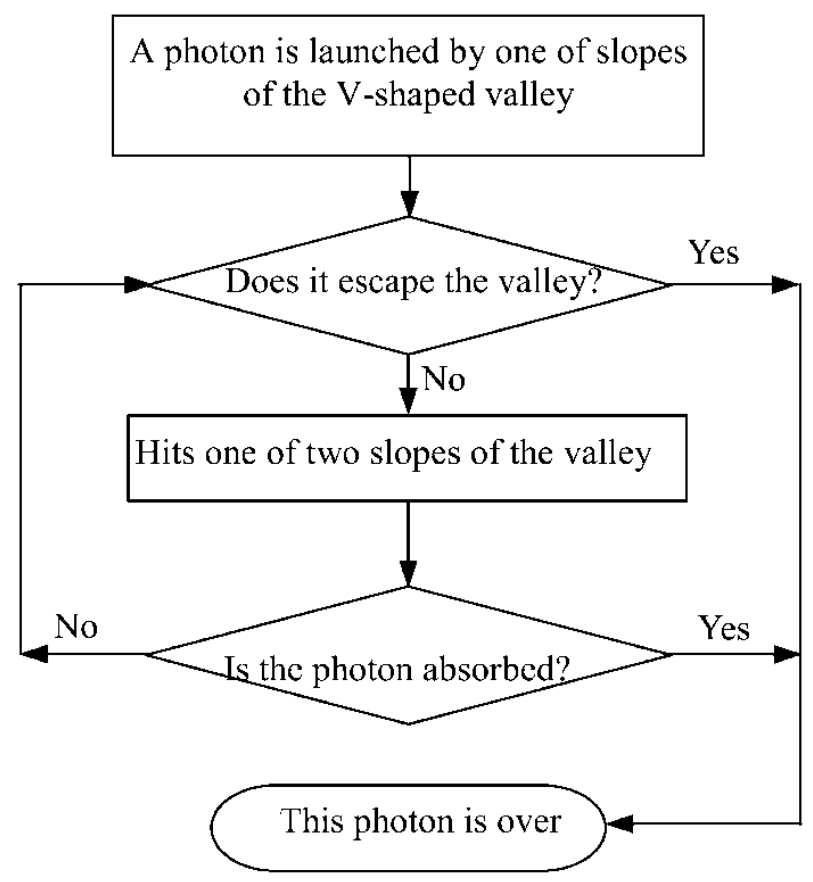

Figure 5. Workflow of launching and tracing a photon. 


$$
p(\mu)=\frac{B_{\lambda}\left(T_{\text {pixelbright }}(\mu)\right)}{B_{\lambda}\left(T_{0}\right)}
$$

In fact, $p(\mu)$ is the effective emissivity of the $\mathrm{V}$-shaped valley obtained by the Monte Carlo simulation.

6. We also calculate the scale effect factor $g(\mu)$ (Li et al. 2000) and compare it with $g(\mu)$.

\subsection{Experiments}

We simulate four thermal emissions from the $\mathrm{V}$-shaped valley with $0.5 \mathrm{~m}$ depth (since the open is $1 \mathrm{~m}$, the bevel of the valley is $45^{\circ}$ ) by four combinations of different material emissivities (Snyder et al. 1998) ( $\left.\varepsilon_{\text {left }}, \varepsilon_{\text {right }}\right)$ and temperatures $\left(T_{\text {left }}, T_{\text {right }}\right)$.

1. The two slopes with the same emissivity but different temperatures:

$$
\varepsilon_{\text {left }}=0.96 ; \varepsilon_{\text {right }}=0.96 ; T_{\text {left }}=290 \mathrm{~K} ; T_{\text {right }}=310 \mathrm{~K}
$$

2. The two slopes with different emissivities and the same temperature:

$$
\varepsilon_{\text {left }}=0.85 ; \varepsilon_{\text {right }}=0.96 ; T_{\text {left }}=300 \mathrm{~K} ; T_{\text {right }}=300 \mathrm{~K}
$$

3. The emissivities and temperatures change with a positive correlation in the two slopes:

$\varepsilon_{\text {left }}=0.85 ; \varepsilon_{\text {right }}=0.96 ; T_{\text {left }}=290 \mathrm{~K} ; T_{\text {right }}=310 \mathrm{~K}$

4. The emissivities and temperatures change with a negative correlation in the two slopes:

$\varepsilon_{\text {left }}=0.85 ; \varepsilon_{\text {right }}=0.96 ; T_{\text {left }}=310 \mathrm{~K} ; T_{\text {right }}=290 \mathrm{~K}$

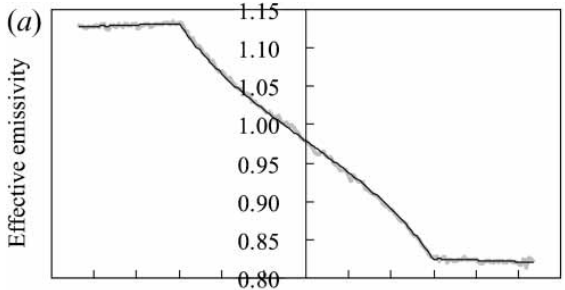

$\begin{array}{lllllllllllll}-90 & -75 & -60 & -45 & -30 & -15 & 0 & 15 & 30 & 45 & 60 & 75 & 90\end{array}$

Viewing zenith angle $\left({ }^{\circ}\right)$

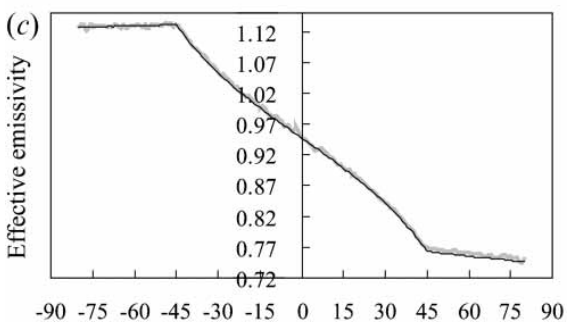

Viewing zenith angle $\left({ }^{\circ}\right)$

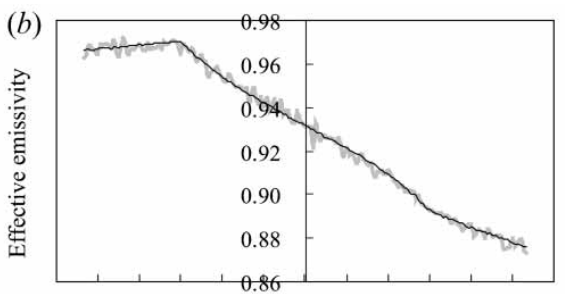

$\begin{array}{lllllllllllll}-90 & -75 & -60 & -45 & -30 & -15 & 0 & 15 & 30 & 45 & 60 & 75 & 90\end{array}$

Viewing zenith angle $\left({ }^{\circ}\right)$

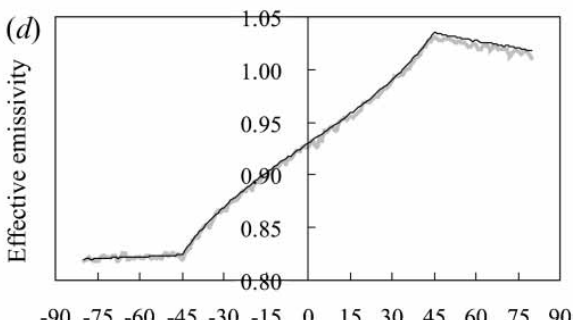

Viewing zenith angle $\left({ }^{\circ}\right)$

Figure 6. Comparison of the simulated and the modelled effective emissivities. (a) Case 1the two slopes with same emissivities and different temperatures. (b) Case 2- the two slopes with different emissivities and same temperatures. (c) Case 3-the emissivities and temperatures change with positive correlation in the two slopes. $(d)$ Case 4 the emissivities and temperatures change with negative correlation in the two slopes. 
The pixel reference temperature $T_{0}$ is defined as $300 \mathrm{~K}$ for computing $p(\mu)$ and $g(\mu)$ in the four simulations. The results of comparing $p(\mu)$ with $g(\mu)$ are shown in figure 6. Assuming that the principal plane is across the plane of the V-shaped valley and the zenith angle for the left wing of the principal plane is positive. The thick grey line represents the simulated values $p(\mu)$, the thin black line represents the modelled values $g(\mu)$. It is very clear that both are matched very well for all cases.

\section{Conclusion and discussion}

In this article, we have first developed the Monte Carlo approach for simulating thermal emission from the pixels of non-isothermal three-dimensional structures, and then deduced an analytical expression of the effective emissivity of the $\mathrm{V}$-shaped valley. The error limit of the analytical expression has also been given.

The V-shaped valley may not represent natural surfaces very well. Due to its simplicity, it is helpful for us to understand how scale effect and directional pattern of thermal emission of non-isothermal heterogeneous surfaces depend on material emissivity, component temperature and the geometry of the surfaces. In reality, the furrows of ploughed soil and the open space of the row crop usually can be treated as approximately V surfaces. Our simulations show that scaling effects of thermal emission from non-isothermal surfaces does exist. So Planck's law should be corrected so that land surface temperatures can be correctly obtained in a remote sensing context, due to the heterogeneous non-isothermal property of remote sensing pixels. Li's model explains the mechanism that gives rise to directional dependence in thermal emissions from non-isothermal surfaces. It is helpful to develop application-specific models to obtain accurate land surface temperature.

The Monte Carlo approach is based on photon transport in pixels. Because Kirchhoff's law is valid only under isothermal conditions, for the sake of simulating thermal emission of the non-isothermal surfaces, photons are transmitted and scattered by these surfaces in this approach. Hence effective directional emissivity of the pixel can be calculated by counting the number of escaped photons in various directions. The Monte Carlo approach may also be used under other nonisothermal conditions.

\section{Acknowledgments}

This work was funded under China's Key-importance Basic Research Program (95-Y-38), the Special Funds for Major State Basic Research Project (G20000779), CNSF-49971059 and US NASA NAG5-7217, NAG5-31369.

\section{References}

BECKER, F., and LI, Z., 1995, Surface temperature and emissivity at various scales: definition, measurement and related problems. Remote Sensing Reviews, 12, 225-253.

Friedl, M. A., Davis, F. W., Michaelsen, J., and Moritz, M. A., 1995, Scaling and uncertainty in the relationship between the NDVI and land surface biophysical variables: an analysis using a scene simulation model and data from FIFE. Remote Sensing of Environment, 54, 223-246.

Li, X., Ni, W., Hu, B., Woodcock, C., and Strahler, A. H., 1996, Decoupling pathscattering of light in a homogeneous layer and multiple bouncing at its nonLambertian bottom. Science in China (Series E), 39, 92-105.

Li, X., Strahler, A. H., and Friedl, M., 1999a, A conceptual model for effective 
directional emissivity from nonisothermal surface. IEEE Transactions on Geoscience and Remote Sensing, 37, 2508-2517.

Li, X., Wang, J., and Strahler, A. H., 1999b, Scale effects of Planck law over a nonisothermal blackbody. Science in China (Series E), 42, 652-656.

Li, X., WANG, J., and Strahler, A. H., 2000, Scale effects and scaling-up by geometricoptical model. Science in China (Series E), 43 (Suppl.), 17-22.

MARCEAU, D. J., and HAY, G. J., 2000, Remote sensing contributions to the scale issue. Canadian Journal of Remote Sensing, 25, 357-366.

Norman, J., and BECKER, F., 1995, Terminology in thermal infrared remote sensing of natural surface. Remote Sensing Reviews, 12, 159-173.

Quattrochi, D. A., 1995, Spatial and temporal scaling of thermal infrared remote sensing data. Remote Sensing Reviews, 12, 255-286.

Quatrochi, D. A., and Goodchild, M. F., eds, 1997, Scale in Remote Sensing and GIS (Boca Raton, FL: CRC Lewis Publishers).

RAFFY, M., 1994, Change of scale theory: a capital challenge for space observation of earth. International Journal of Remote Sensing, 15, 2353-2357.

SNYDER, W. C., WAN, Z., ZHANG, Y., and FENG, Y. Z., 1998, Classification-based emissivity for land surface temperature measurement from space. International Journal of Remote Sensing, 19, 2753-2774.

Su, H-B., Zhang, R-H., TANG, X-Z., and Sun, X-M., 2000, Determination of effective emissivity for the regular and irregular cavities using Monte-Carlo method. International Journal of Remote Sensing, 21, 2313-2320.

WAN, Z., and DoIZER, J., 1996, A generalized split-window algorithm for retrieving landsurface temperature from space. IEEE Transactions on Geoscience and Remote Sensing, 34, 892-905.

Woodcock, C. E., and Strahler, A. H., 1987, The factor of scale in remote sensing. Remote Sensing of Environment, 21, 311-332. 